\title{
news feature
}

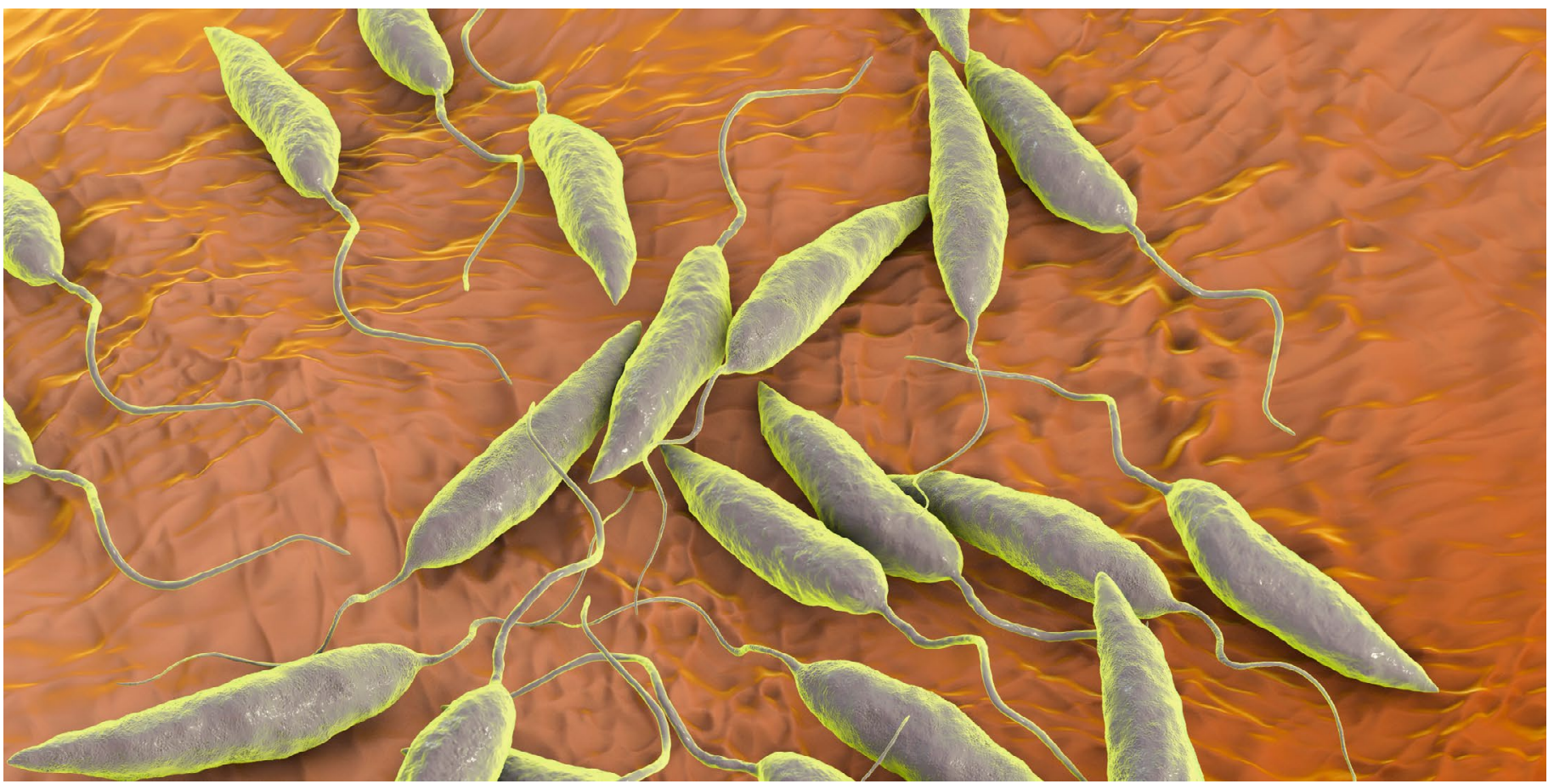

\section{1 clinical trials that will shape medicine in 2022}

Nature Medicine asks leading researchers to name their most anticipated clinical trial for 2022, from CRISPR and psilocybin to oncolytic viruses.

\section{Carrie Arnold}<smiles>C1C2CC3CC1C3C2</smiles>
espite the ongoing disruptions from COVID-19, 2021 saw some major results from clinical trials.

2022 looks to be a bumper year too, so Nature Medicine asks leaders in their field what they think will be the most important clinical trial results in the coming year.

\section{Universal influenza vaccines}

Neil P. King: Like existing vaccines against seasonal influenza, FluMos-v1 (Table 1) is meant to create antibodies to several different influenza virus strains. Unlike existing influenza shots, the new vaccine uses a mosaic nanoparticle immunogen that co-displays antigens from multiple influenza viruses on the same nanoparticle surface. In our pre-clinical work, we found that the nanoparticle immunogen elicited a greater diversity of protective antibodies than did current vaccines against influenza. We are particularly interested in learning about how the vaccine will interact with immune systems that have encountered influenza viruses or vaccines before. This pre-existing immunity may alter vaccine performance, and this is something we couldn't get a read on pre-clinically.

Neil P. King is an assistant professor at the Institute for Protein Design, University of Washington.

\section{Antisense oligonucleotides for Huntington's}

Claudia Testa: This trial for Huntington's disease closed early, due to futility, in March 2021. Despite this setback, the full results are not yet out and are critically important. This antisense oligonucleotide (ASO) targets both mutant and wild-type huntingtin mRNA, which is completely different from other ASOs on the market. The trial results will be a first real look at fundamental aspects of
RNA-lowering strategies, including how the drug impacted various biomarkers across hundreds of participants. The results could transform the clinical-trial landscape for other conditions treatable by ASOs. This trial perfectly illustrates how well-designed and well-run trials do not entirely fail - the efforts of the trial participants will be crucial to accelerating the timeline to a safe and effective Huntington's disease-modifying treatment, even though the trial outcome will not lead to an agent approved by the US Food and Drug Administration.

Claudia Testa is the division chief of Precision Medicine and Neurogenetics, UNC Chapel Hill.

\section{CRISPR for amyloidosis}

Julian Gillmore: I have been looking after patients with transthyretin amyloidosis for 25 years, and until 5 years ago, I just watched 
Table 1 | Clinical trials in 2022

\begin{tabular}{|c|c|c|c|}
\hline Treatment & Condition or disease & Sponsor & Phase and size \\
\hline $\begin{array}{l}\text { Mosaic nanoparticle } \\
\text { immunogen FluMos-v1 }\end{array}$ & Influenza & NIAID & Phase 1; 35 participants \\
\hline ASOs & Huntington's disease & Roche / Genentech & Phase 3; 791 participants \\
\hline $\begin{array}{l}\text { CRISPR-based gene therapy } \\
\text { NTLA-2001 }\end{array}$ & Transthyretin amyloidosis & Regeneron / Intellia Therapeutics & Phase 1; 38 participants \\
\hline Miltefosine and paromomycin & Visceral leishmaniasis (kala-azar) & Drugs for Neglected Diseases Initiative & Phase 3; 439 participants \\
\hline Pridopidine & Huntington's disease & Prilenia Therapeutics & $\begin{array}{l}\text { Phase } 2 / 3 \text {; up to } 480 \\
\text { participants }\end{array}$ \\
\hline AAV9 U7 snRNA gene therapy & Duchenne muscular dystrophy & $\begin{array}{l}\text { Nationwide Children's Hospital / Audentes } \\
\text { Therapeutics }\end{array}$ & Phase 1/2a; 3 participants \\
\hline Psilocybin & Treatment-resistant depression & COMPASS Pathways & Phase 2a; 231 participants \\
\hline $\begin{array}{l}\text { Oncolytic vaccinia virus } \\
\text { ASP9801 }\end{array}$ & Advanced or metastatic solid tumors & Astellas & Phase 1; 105 participants \\
\hline $\begin{array}{l}\text { Monoclonal antibody } \\
\text { nirsevimab }\end{array}$ & RSV & Medlmmune / AstraZeneca & Phase 3, 3000 participants \\
\hline $\begin{array}{l}\text { Monoclonal antibody } \\
\text { pembrolizumab }\end{array}$ & Triple negative breast cancer & National Cancer Institute & Phase 3; 1155 participants \\
\hline Various & COVID-19 & TOGETHER Trial & Adaptive trial; various \\
\hline
\end{tabular}

NIAID, National Institute of Allergy and Infectious Diseases; AAV9, adeno-associated virus 9; snRNA, small nuclear RNA.

their health decline. Some years ago, gene silencers became available and improved outcomes for patients, which was a very substantial advance. But patients on gene silencers need lifelong repeated treatments, and although most patients' disease stabilizes, some continue to deteriorate. Preliminary data from this trial have shown for the first time that CRISPR-based gene therapies can be administered by intravenous infusion to edit a specific gene in liver cells. Full results are expected next year. This new gene-editing therapy offers patients the prospect of meaningful clinical improvements after a single dose of the drug.

Julian Gillmore is a reader in medicine and an honorary consultant nephrologist at Royal Free London Hospital.

\section{Oral therapy for kala-azar}

Monique Wasunna: I am excited to see the results of our phase 3 clinical trial looking for a better regimen to treat visceral leishmaniasis, also known as 'kala-azar'. This trial tests new combination regimens of miltefosine and paromomycin in Ethiopia, Sudan, Kenya and Uganda in eastern Africa. The aim is to replace the toxic sodium stibogluconate component of the current injectable treatment for kala-azar with the orally administered miltefosine. This is a much-needed improvement patients have been waiting for and we have been working toward for years, as oral treatments can be administered at local health centers, closer to affected communities. Patients diagnosed with kala-azar will die if they are not treated. The new, less toxic and more easily administered regimen will also be good for children, who are disproportionately affected by visceral leishmaniasis. Children under 12 represented $60 \%$ of clinical-trial patients, and preliminary results show a promisingly high efficacy for them.

Monique Wasunna is the director of the Drugs for Neglected Diseases Initiative Africa Regional Office, Nairobi.

\section{Targeting sigma 1 receptor in neurodegenerative disease} Blair Leavitt: There are several ongoing studies of pridopidine that target disease progression in neurodegenerative disorders. Pridopidine is an oral small-molecule therapeutic that selectively targets the sigma 1 receptor and has a well-established safety record from previous human clinical trials. Over the past several years, a role for sigma 1 receptor in neurodegeneration has been established, and there has been quite a bit of promising pre-clinical data to suggest that pridopidine has protective effects in Huntington's disease and in other neurodegenerative disorders such as amyotrophic lateral sclerosis. Pridopidine is the only drug being tested in a phase 3 clinical trial that will assess the clinical progression of Huntington's disease. There is an important phase $2 / 3$ trial of pridopidine in amyotrophic lateral sclerosis that is also currently underway, and the results of these ongoing studies are eagerly awaited by the community.
Blair Leavitt is a consulting neurologist and director of research at the University of British Columbia Centre for Huntington's Disease.

\section{Exon skipping in muscular dystrophy} Kevin M. Flanigan: A small number of boys with Duchenne muscular dystrophy have a disease caused by a duplication of exon 2 of the DMD gene (which encodes dystrophin). This exon duplication can be removed through the use of an adenoassociated virus 9-based vector, which carries copies of the non-coding small nuclear RNA U7 with a sequence directed toward splice donor and acceptor sites within the duplicated exon. This exonskipping approach is a first in-human trial and results in exclusion of a copy of DMD exon 2, and expression of the full-length, wild-type dystrophin. Preliminary data have been presented showing successful expression, for the first time, of full-length dystrophin in response to this viral gene therapy. There are four more gene therapies for Duchenne muscular dystrophy being tested, but each of these requires delivery of a gene encoding an engineered microdystrophin, which is not present in nature and which is expected to result in substantial functional improvement.

Kevin M. Flanigan is an attending neurologist at Nationwide Children's, the Robert F. and Edgar T. Wolfe Foundation Endowed Chair in Neuromuscular Research and a professor of pediatrics and neurology at The Ohio State University College of Medicine. 


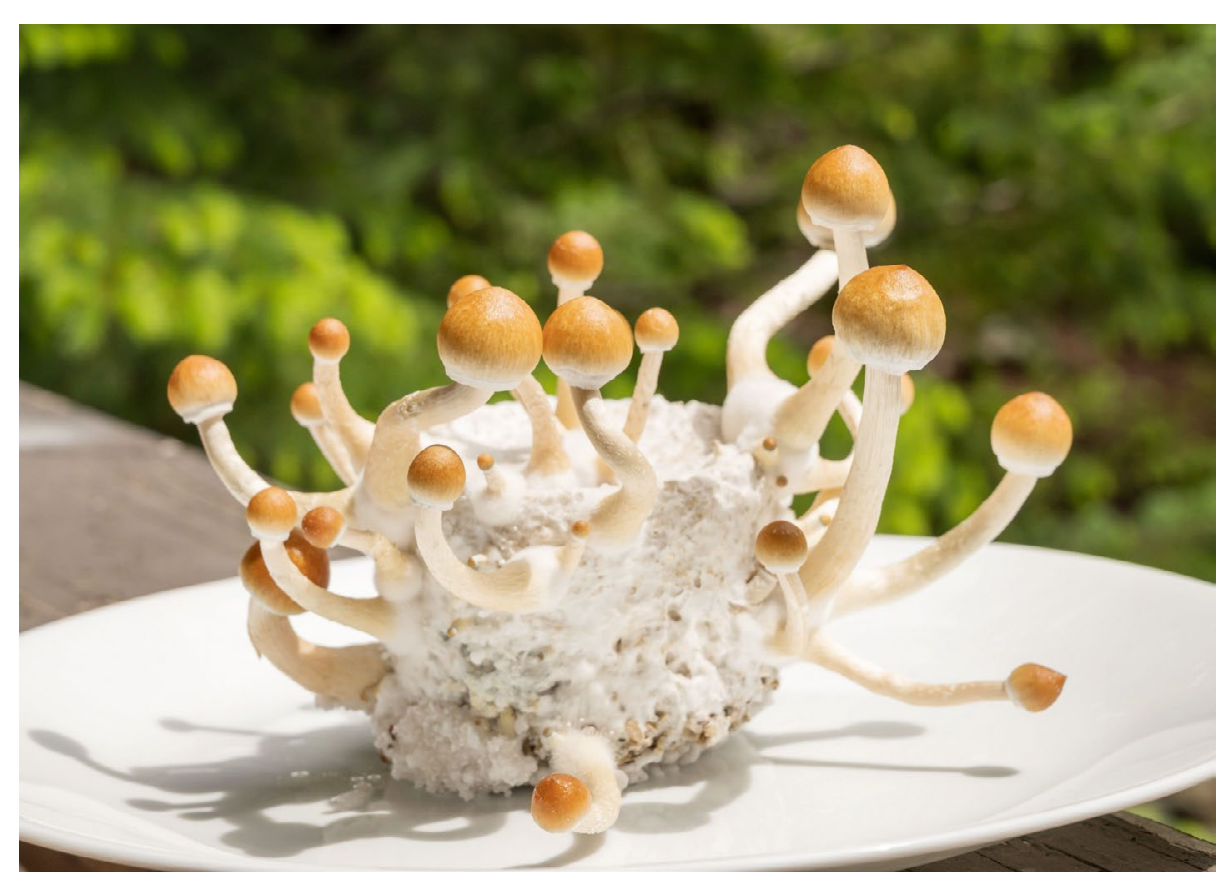

Psilocybin is being tested for treatment-resistant depression. Credit: David Buzzard / Alamy Stock Photo.

\section{Psychedelic therapy}

David Nutt: Psilocybin, a psychedelic derived from fungi, is showing great promise in the treatment of mental-health conditions. I'm excited about this psilocybin trial for three main reasons. First, it tackles the huge problem of depression that is resistant to existing treatments. Second, it's a multi-center and multi-country trial, which will let researchers test the psychedelic therapy in a diverse group of people. Finally, it will also give some readouts on the doseeffect relationship, as they are testing three greatly differing doses of psilocybin, at $1 \mathrm{mg}$, $10 \mathrm{mg}$ and $25 \mathrm{mg}$.

David Nutt is the Edmond J. Safra Professor of Neuropsychopharmacology and director of the Neuropsychopharmacology Unit in the Division of Brain Sciences, Imperial College London.

\section{Oncolytic viruses}

Yoshikazu Yonemitsu: This is a phase 1 open-label study of ASP9801 administered by intratumoral injection in patients with advanced or metastatic solid tumors. ASP9801 is a genetically engineered oncolytic vaccinia virus that expresses two cytokines, IL-7 and IL-12, that help stimulate antitumor immune responses. ASP9801 was effective in multiple immunocompetent mouse models and showed promising results in both directly treated tumors and distant tumors.

This study will for the first time assess the safety, tolerability and antitumor activity of this oncolytic vaccinia virus in patients with cancer.

\section{Yoshikazu Yonemitsu is a professor in the} Graduate School of Pharmaceutical Sciences, Kyushu University.

\section{Long-lasting monoclonal antibodies for RSV}

Ruth A. Karron: Respiratory syncytial virus (RSV) is a leading cause of severe respiratory infections in infants and children, but there is currently no vaccine against this. Nirsevimab is a monoclonal antibody that targets the F protein on the virus's surface. It carries a YTE mutation, which extends its half-life; this means that a single dose of the antibody could protect an infant throughout their first RSV season. This is a big improvement over the current monoclonal antibody to RSV, palivizumab, which lasts about a month and therefore is used only for high-risk infants. The final results will be interesting, because they will determine if vaccine-policymaking bodies are willing to approve a monoclonal antibody, rather than a vaccine, as a prophylactic product.

\section{Ruth A. Karron is a professor in the} Department of International Health, Johns Hopkins Bloomberg School of Public Health, the director of the Center for Immunization Research, and the founding director of the Johns Hopkins Vaccine Initiative.

\section{Adjuvant immunotherapy for breast cancer} Jennifer Litton: People with triplenegative breast cancer have fewer treatment options than others with this type of cancer. Although pembrolizumab (marketed as Keytruda) was originally developed to treat advanced melanoma, it has since been approved to treat a variety of cancers. This trial will answer important questions about the use of adjuvant pembrolizumab for patients with high-risk triple-negative breast cancer. The Keynote 522 study provided important information about the use of neoadjuvant pembrolizumab for these patients, but it also left many questions about the length of treatment and the role of this drug in the post-operative space. This study will shed important light on these questions as a guide for moving forward.

\section{Jennifer Litton is the vice president of} clinical research and a professor in the Breast Medical Oncology department, MD Anderson Cancer Center.

\section{COVID-19 treatments}

Edward Mills: The TOGETHER trial has been groundbreaking, as it has evaluated nine different interventions in 2021 and managed to drop six of them very early due to lack of efficacy, thereby saving time and money. Most importantly, and what I am most excited about, TOGETHER is the first trial to demonstrate that a low-cost repurposed medicine, fluvoxamine, can prevent hospitalizations for COVID-19. We are now moving into combinations of drugs, and I think that is where the future of COVID-19 treatment is. In the next year, we should learn about the efficacy of several combinations, including fluvoxamine or fluoxetine plus an inhaled steroid, as well as fluvoxamine plus the direct-acting antiviral molnupiravir.

Edward Mills is a professor of health sciences at McMaster University.

Editorial note These interviews have been edited for length and clarity.

Carrie Arnold

Science writer, Richmond, VA, USA.

Published online: 10 December 2021 https://doi.org/10.1038/s41591-021-01601-5 